\title{
Wie Covid-19 meine Arbeit verändert hat
}

\author{
Iris Najjar
}

Assistenzärztin in Allgemeiner Innerer Medizin, Hôpitaux universitaires de Genève

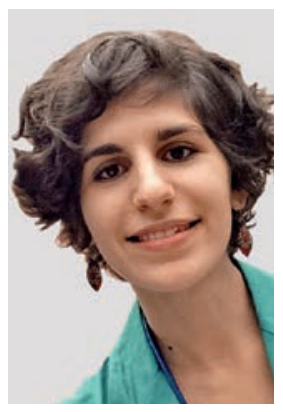

«Hallo, Iris! Wie geht's dir denn so? Ich hoffe gut. Wir sind bei dir, Kopf hoch!» Seit die Corona-Epidemie Europa heimsucht, erhalte ich täglich aufmunternde Botschaften aus dem Familien- und Freundeskreis, sogar von längst aus dem Blick geratenen Bekannten. Vor der Krise war mein beruflicher Alltag nicht allzu aufregend: keine Reanimationen und keine Szenen à la Dr. House, sondern 12-Stunden-Tage, von denen ein grosser Teil auf Verwaltungsarbeit entfiel. Wir haben uns daran gewöhnen müssen, dass diese administrativen Aufgaben die Zeit von Ärztinnen und Ärzten massiv in Beschlag nehmen: allerlei Schreiben verfassen; Überweisungen an andere Einrichtungen organisieren; die an der Behandlung beteiligten Fachärzte und Allgemeinmediziner koordinieren ... die meiste Zeit verbringen wir mittlerweile am Bildschirm oder Telefon. Dazu die Gespräche mit den Patienten und ihren Familien: emotional, zeitaufwendig und doch so wichtig. Alles so eng getaktet, dass kaum Zeit zum Essen bleibt. Und dennoch habe ich bis vor wenigen Wochen nie eine Botschaft erhalten, die mir gesendet wurde, um mir bei dieser schwierigen Arbeit Mut zu machen. Nach nicht einmal einer Woche stehen uns plötzlich zusätzliche medizinische und pflegerische Kräfte zur Seite, dazu Bereitschaftspersonal. Die klinische Dokumentation ist auf das absolute Minimum beschränkt und teilweise automatisiert. Überweisungen an andere Einrichtungen erfolgen schnell und effizient. Dies sind nur einige Beispiele für die Veränderungen während der Krise, die unsere Arbeitsbedingungen deutlich verbessert haben. Und erst seit wegen der Ansteckungsgefahr ein Besuchsverbot besteht, ist mir klar, wie sehr die Gespräche mit den Familien mich emotional mitgenommen haben. Plötzlich können wir sogar in Ruhe zu Mittag essen.

Es ist paradox: Es bedurfte einer Pandemie, um Verbesserungen in unserem Arbeitsumfeld zu bewirken, die wir nie für möglich gehalten hätten.

\section{Und wenn die Krise überstanden ist?}

Wir müssen die derzeitige Dynamik nutzen, um diese Veränderungen fortzuführen und zu vertiefen. Eine weitere Automatisierung und Vereinfachung der klinischen
Dokumentation scheint mir unabdingbar, damit wir mehr Zeit am Krankenbett verbringen und uns so wieder auf den Kern unseres Berufs konzentrieren können. Schon vor dem Coronavirus waren wir an unsere Grenzen gestossen. Es ist unerlässlich, einen Teil des während der Krise zusätzlich eingestellten Personals zu behalten. Zwar haben wir in normalen Zeiten weniger Patientinnen und Patienten zu versorgen, dafür aber mit deutlich komplexeren und multimorbiden Krankheitsbildern. Eine Aufstockung des Personals würde zum einen die medizinischen Teams entlasten und die Patientenversorgung verbessern, zum anderen wären wir in der Einteilung unserer Zeit flexibler: Teilzeitarbeit würde uns mehr Zeit für unsere Familien geben, die Reduzierung der Überstunden würde eine gesündere Work-Life-Balance ermöglichen, und neben der klinischen Arbeit könnten wir Forschung betreiben. All dies war vor der Krise schwierig, wenn nicht unerreichbar. Ich bin mir bewusst, dass ich diese Forderungen in einer durchaus privilegierten Position vorbringe - als Ärztin in der Schweiz. Die angesprochenen Probleme sind jedoch in den meisten Ländern mit entwickelten Gesundheitssystemen anzutreffen und verschärfen sich tendenziell. Diese Krise führt uns allen vor Augen, welch entscheidende Rolle die Gesundheit dabei spielt, dass es unserer Gesellschaft gut geht. In der Westschweiz treten die Menschen jeden Abend auf ihre Balkone und applaudieren dem medizinischen Personal. Es ist bewegend, diese Unterstützung zu spüren. Gerade für die in der Pflege arbeitenden Menschen und damit ihre Familien besteht eine hohe Gefahr der Ansteckung mit dem Coronavirus. Aber auch in «normalen Zeiten» birgt unser Beruf Risiken: saisonale Grippe, Magen-Darm-Grippe, Kontakt zu Patienten mit multiresistenten Keimen - ganz zu schweigen von verbalen und körperlichen Angriffen.

Es musste erst das Coronavirus kommen, damit der Allgemeinheit bewusst wurde, welchen Risiken wir bei der Arbeit in diesem Umfeld ausgesetzt sind.

Dieses neue Bewusstsein hat eine Welle der Solidarität ausgelöst, bis hin zu Bankern, die nun die Mahlzeiten des Spitalpersonals sponsern. Es ist an der Zeit, diese kurzzeitig aufgeflammte Solidarität in einen Systemwandel münden zu lassen. 